An official journal of the / Un journal officiel de la "Société Sénégalaise de Cancérologie" (SOSECAN)

Journal homepage: www.africanjournalofoncology.com

Original article / Article original

DOI: https://doi.org/I0.54266/ajo.I.I.6.6

\title{
Assessment of prognostic factors in breast cancer in women in Senegal: a review of 288 cases
}

\section{Evaluation des facteurs pronostiques dans le cancer du sein chez la femme au Sénégal : à propos de 288 cas}

\author{
M. Ndiaye', S. Dieng', J. Thiam', AC. Diallo', D. Diouf', S. Ka', A. Dem'. \\ ' Institut Joliot Curie de Dakar, Sénégal.
}

\begin{abstract}
INTRODUCTION: In Senegal, breast cancer is the second female cancer and poses a major public health problem. The aim of this work was to assess prognostic factors in the progression of breast cancer in women. PATIENTS AND METHODS: This is a retrospective study carried out over a period of one year from January I, 2008 to December 3I, 2008 on all the women followed for breast cancer in the oncology department at the Joliot Curie Institute. Thus, 288 breast cancer patients were collected. RESULTS: The average age of our patients was 47.32 years. The average parity was 4.9 children per woman. Twenty-two or $7 \%$ of patients had a history of cancer. Clinically, the tumor size was classified as T4 in 180 patients, ie $81 \%$. Lymph node involvement in 188 patients (65.2\%). The most frequent histological type was invasive ductal carcinoma with $90.3 \%$ of cases. A predominance of grades SBRII and SBRIII was observed (respectively $41 \%$ and $46 \%$ ). Hormone receptors (RH) were positive in eight cases (24\%). Overexpression of the HER2 gene was found in only four out of 30 patients. The limits of the surgery were specified in 48 patients with invaded margins in seven patients (14.5\%). The presence of vascular emboli was noted in 18 patients among the 29 whose research was carried out in 179 patients, ie $62 \%$. At the time of the initial diagnosis, 45 patients or $19.7 \%$ of patients presented at least one distant metastasis. The majority of patients were received at an advanced stage (89\%, classified between stage III and IV). Only one patient was received at stage I. Overall survival for breast cancer was $72 \%$ at 3 years and $30 \%$ at 5 years. The 5 -year overall survival of patients with localized disease was $85 \%$ compared to $5 \%$ for patients with advanced stage. CONCLUSION: The prognostic factors are multiple and often pejorative in our patients with a predominance of young women, locally advanced cancers and aggressive biological forms.
\end{abstract}

KEYWORDS: Breast cancer; Grade SBR; Hormone receptors; HER2.

INTRODUCTION : Au Sénégal le cancer du sein représente le deuxième cancer féminin et pose un problème de santé public majeur. Le but de ce travail était d'évaluer les facteurs pronostiques dans l'évolution du cancer du sein chez la femme. PATIENTS ET METHODES : II s'agit d'une étude rétrospective réalisée sur une période sur un an allant du I janvier 2008 au 3 I Décembre 2008 portant sur l'ensemble des femmes suivies pour cancer du sein au service de cancérologie à l'Institut Joliot Curie. Ainsi, 288 patientes atteintes de cancer du sein ont été colligés. RESULTATS : La moyenne d'âge de nos malades était de 47,32 ans. La parité moyenne était de 4,9 enfants par femme. Vingt-deux soit 7\% des patientes avaient un antécédent de cancer. Cliniquement, la taille tumorale était classée T4 chez 180 patientes soit $81 \%$. Une atteinte ganglionnaire chez 188 patientes (65,2\%). Le type histologique le plus fréquent était le carcinome canalaire infiltrant avec $90,3 \%$ des cas. Une prédominance des grades SBRII et SBRIII a été observé (respectivement $41 \%$ et $46 \%)$. Les récepteurs hormonaux $(\mathrm{RH})$ étaient positifs dans huit cas $(24 \%)$. La surexpression du gène HER2 était retrouvée chez seulement quatre patientes sur 30. Les limites de la chirurgie étaient précisées chez 48 patientes avec des marges envahies chez sept patientes (14,5\%). La présence d'emboles vasculaires était notée chez 18 patientes parmi les 29 dont la recherche a été effectuée chez 179 patientes soit $62 \%$. Au moment du diagnostic initial, 45 patientes soit $19,7 \%$ des malades présentaient au moins une métastase distance. La majorité des patientes étaient reçues à un stade avancé (89\%, classées entre stade III et IV). Une seule patiente était reçue au stade I. La survie globale pour les cancers du sein était à $72 \%$ à 3 ans et $30 \%$ à 5 ans. La survie globale à 5 ans des patientes au stade localisé de la maladie était de $85 \%$ contre $5 \%$ pour les patientes au stade avancé. CONCLUSION : Les facteurs pronostiques sont multiples et souvent péjoratifs chez nos patients avec une prédominance des femmes jeunes, des cancers localement avancés et des formes biologiques agressifs.

MOTS-CLES : Cancer du sein ; Grade SBR ; Récepteurs hormonaux ; HER2.

\section{INTRODUCTION}

Le cancer du sein est actuellement la tumeur maligne la plus fréquente chez la femme dans le monde et la deuxième au Sénégal. II reste une maladie grave qui est en augmentation régulière. Ainsi l'étude sur les facteurs pronostiques serait d'un grand apport pour une prise en charge adéquate des patientes qui demeure pluridisciplinaire. Le but de ce travail est d'évaluer la prévalence des facteurs pronostiques dans l'évolution du cancer du sein chez la femme au Sénégal.

\section{PATIENTS ET METHODES}

II s'agit d'une étude rétrospective réalisée sur une période sur un an allant du $I^{\mathrm{er}}$ janvier 2008 au 3 I Décembre 2008 portant sur l'ensemble des femmes suivies pour cancer du sein au 
service de cancérologie à l'Institut Joliot Curie. Ainsi, 288 patientes atteintes de cancer du sein ont été colligés.

\section{RESULTATS}

La moyenne d'âge de nos malades était de 47,32 ans. La parité moyenne était de 4,9 enfants par femme. Cent trente-sept soit $49 \%$ des patientes étaient ménopausées. Trente-trois patientes soit II\% avaient utilisé une contraception hormonale. Vingtdeux soit $7 \%$ des patientes avaient un antécédent de cancer. La masse du sein était le principal motif de consultation chez 211 de nos patientes soit $76,7 \%$. Cliniquement, la taille tumorale était classée T4 chez 180 patientes soit $81 \%$. Une atteinte ganglionnaire chez 188 patientes (65,2\%). Le type histologique le plus fréquent était le carcinome canalaire infiltrant avec $90,3 \%$ des cas. Une prédominance des grades SBRII et SBRIII a été observé (respectivement 4 I\% et $46 \%$ ) (Tableau I).

Tableau I : Répartition des patientes en fonction du grade histopronostiques SBR

\begin{tabular}{|lll|}
\hline SBR & Effectif & Pourcentage (\%) \\
\hline I & 19 & 12 \\
II & 65 & 4 I \\
III & 74 & 46 \\
Total & 158 & 100 \\
\hline
\end{tabular}

Les récepteurs hormonaux $(\mathrm{RH})$ étaient positifs dans huit cas (24\%). La surexpression du gène HER2 était positive chez seulement quatre sur 30 patientes. Les limites de la chirurgie étaient précisées que chez 48 patientes avec des marges envahies chez sept patientes. La présence d'emboles vasculaires était notée chez 18 patientes parmi les 29 dont la recherche a été effectuée chez 179 patientes soit $62 \%$. Au moment du diagnostic initial, 45 patientes soit 19,7\% des malades présentaient au moins une métastase distance. Le siège des métastases synchrones était ganglionnaire dans $38 \%$, pulmonaire dans $26 \%$ rarement vers les autres organes (foie, os, cerveau, cutanée). La majorité des patientes étaient reçues à un stade avance $(89 \%$, classées entre stade III et IV) (Figure I).

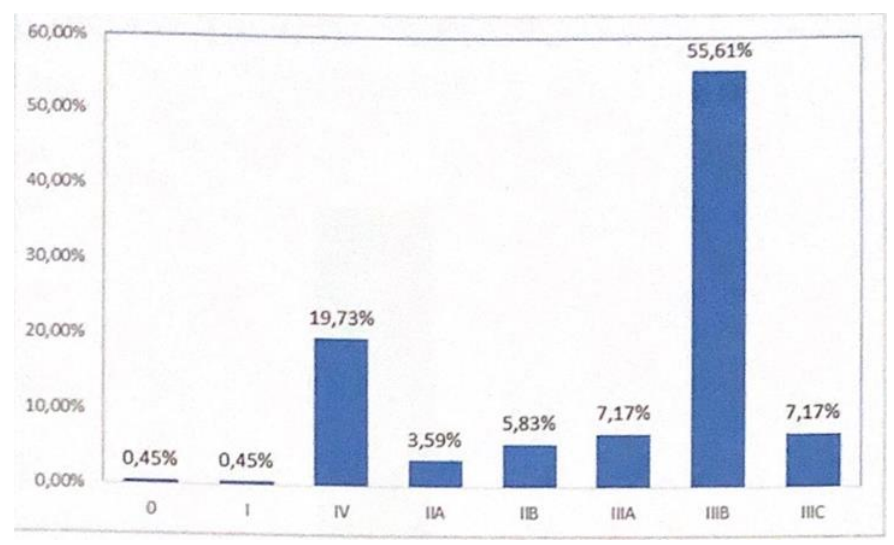

Figure I : Répartition des patientes en fonction de leur stade clinique selon la classification TNM.

Une seule patiente était reçue au stade I. Le délai moyen de suivi était de 20,42 mois chez les patientes. Une récidive était retrouvée chez $10 \%$ de nos patientes avec un délai moyen d'apparition de la récidive de 33,38 mois. Des métastases métachrones étaient notées chez $17 \%$ des patientes avec un siège préférentiel pulmonaire, hépatique, ganglionnaire et osseux. La survie globale pour les cancers du sein était à $72 \%$ à 3 ans et $30 \%$ à 5 ans (Figure 2).

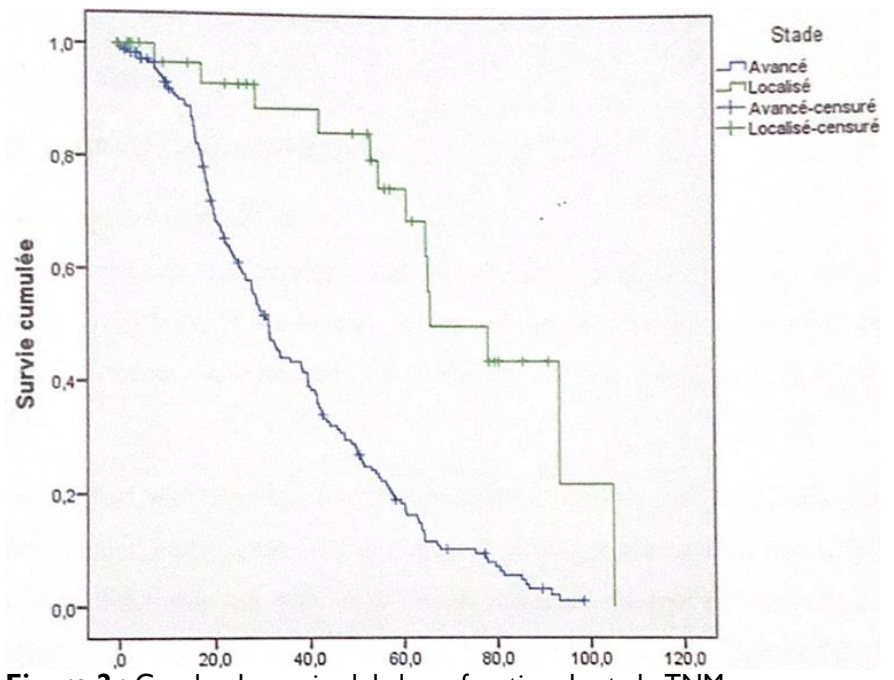

Figure 2 : Courbe de survie globale en fonction du stade TNM.

La survie globale à 5 ans des patientes aux stades localisés de la maladie était de $85 \%$ contre $5 \%$ pour les patientes aux stades avancés.

\section{DISCUSSION}

L'analyse de la littérature montre que l'incidence du cancer du sein augmente avec l'âge, dans notre série jusqu'à un pic situé entre 40 et 49 ans, ce qui était décrit aussi dans l'étude de Dem et al [I]. Ces chiffres sont cependant différents de ceux obtenus dans les études effectuées dans la zone européenne ou l'âge moyen au diagnostic semble être différent de celui de l'Afrique car plus de $50 \%$ des cancers du sein surviennent chez des femmes de 65 ans ou plus et seuls $25 \%$ surviennent avant l'âge de 50 ans avec un âge moyen au diagnostic de $6 \mathrm{I}$ ans [2, 3].

Par ailleurs, la prédominance du cancer au niveau d'un sein par rapport à l'autre s'expliquerait par les habitudes d'allaitement [4]. Le siège de la tumeur était dans 140 des cas (50\%) au niveau du sein droit, contre $47 \%$ au sein gauche. Le cancer était bilatéral chez $3 \%$ des patientes (Sept patientes).

Selon l'étude de Dem et al [I], la plupart des patientes étaient vues tard (56,8 \%) au stade locorégional (10,8\%) en situation métastatique d'emblée. Cette situation semble identique à notre série ou la taille clinique avait été spécifié chez 222 patientes. Nous avons retrouvé deux cas de tumeur classe TI soit $0,90 \%, 13$ cas classe T2 soit 5,86\%, 24 cas de tumeur T3 soit $10,81 \%$ et les T4 étaient les plus représentées avec 180 cas soit $81,09 \%$. Seul un cas de Tis et un cas de Tx ont été noté dans la série.

En outre, de nombreux auteurs s'accordent sur le fait que l'envahissement ganglionnaire est plus fréquemment observé chez la femme jeune. Ainsi, selon Gajdos et al [5], 37\% des femmes jeunes (40 ans) présentent un envahissement ganglionnaire versus $25 \%$ chez les patientes âgées, il a trouvé que le pourcentage de cet envahissement augmente parallèlement avec le jeune âge et aussi le volume tumoral. Kroman et al [4] confirment que les femmes jeunes ont un risque plus élevé d'avoir des métastases ganglionnaires. Ces 
assertions pourraient justifier l'important pourcentage d'atteinte ganglionnaire dans notre étude $(82 \%, n=188)$ ou la population de jeune femme est majoritaire et la moyenne d'âge est égale 47 ans.

Dans notre série, l'étude anatomopathologique était effectuée à partir de prélèvement cytologique chez I33 patientes (48\%) et/ou biopsique chez II I patientes (40\%) tous en faveur de la malignité sans aucune discordance notée entre les types de prélèvements.

Sur le plan histologique, on constate que le carcinome canalaire infiltrant $(\mathrm{CCl})$ est le type histologique le plus fréquent dans le cancer du sein. Ces résultats sont semblables à l'étude de Chauleur et al [6] qui avaient retrouvé $95 \%$ de $\mathrm{CCl}$ et $5 \% \mathrm{CLI}$. Cutuli et al [7] avait retrouvé $83,3 \%$ de carcinome canalaire infiltrant $(\mathrm{CCl}), 22,7 \%$ de carcinome lobulaire infiltrant (CLI), $10 \%$ pour les autres types histologiques. Raymond et al [8] avait retrouvé des résultats semblables avec prédominance du $\mathrm{CCl}(72,9 \%)$ et du CLI (5,2\%).

Le score basé sur certains facteurs détermine les grades I, II et III d'agressivité croissante. Ainsi, le grade III est de plus mauvais pronostic que le grade I et II. C'est aussi un facteur prédictif de réponse à la chimiothérapie [9]. Les tumeurs de grade III répondent mieux à la chimiothérapie que celles classées grade I ou II [9]. Le grade le plus observé dans notre série était le SBR III, noté chez 74 malades, soit $46 \%$, suivi du SBR II dans 65 cas (4I\%), et le SBR I dans I 9 cas, soit I2\%. Ces résultats sont comparables à ceux de Cutuli et al [7] qui avaient retrouvé une prédominance des tumeurs de grade SBR III dans $37,8 \%$ et d'autre part à ceux de Raymond et al [8] qui avaient retrouvé $36,4 \%$ de SBR III. En Europe et aux Etats Unis, ces tumeurs de grade intermédiaire SBR II étaient les plus représentées. Ainsi l'étude ATAC [10] avait retrouvé $46,8 \%$ de SBR II, Punglia et al [II] avait retrouvé 39,3\% de SBR II. Dans notre série, le taux des tumeurs de bon grade histopronostique SBR I (I $\%$ ) était plus faible ainsi Cutuli et al [7] avaient retrouvé $18,3 \%$ et l'étude ATAC [I0] 20,8\%.

Le dosage des récepteurs hormonaux permet de distinguer les tumeurs de bon pronostic avec des RH positifs de celles de mauvais pronostic $\mathrm{RH}$ négatifs. Les récepteurs hormonaux représentent un facteur pronostique et prédictif de la réponse à une hormonothérapie [12]. Dans l'étude de Delozier et al [12], il existerait une corrélation entre l'expression des récepteurs hormonaux et la réponse à la chimiothérapie et les tumeurs qui n'expriment pas les récepteurs hormonaux semblent plus chimio-sensibles. Dans notre série, le dosage des récepteurs hormonaux $(\mathrm{RH})$ était fait que chez 33 patientes ( I $2 \%$ ). II était positif chez huit patientes soit $24 \%$ et négatif chez les 25 autres (76\%). Parmi les patientes $\mathrm{RH}(-)$, la réponse à la chimiothérapie évaluée était partielle avec un seul cas de réponse complète.

L'intérêt de la détermination du statut de I'HER2 dans les carcinomes mammaires est double, par sa valeur pronostique et l'étude de la sensibilité à la chimiothérapie à l'hormonothérapie mais aussi plus récemment pour la thérapie ciblée. En effet les tumeurs HER2(+) étaient plus sensible à ces différents moyens thérapeutiques alors le HER2(-) ont une valeur pronostique péjoratif [9].

Dans notre série, le statut HER2 était précisée chez 30 patientes soit $10 \%$; il était exprimé chez quatre patientes (13\%) et négative pour les 26 autres patientes ( $86 \%$ ).
L'étude de ce paramètre biologique pourrait bien améliorer le pronostic des patientes avec l'avènement de I'hormonothérapie et surtout de la thérapie ciblée [9, 13].

L'étude de la marge d'exérèse de la pièce opératoire est un paramètre important car influant sur le risque de récidive locale, en effet les marges sont considérées comme saines quand elles dépassent $3 \mathrm{~mm}$. C'est un facteur de risque de rechute locale dans les traitements conservateurs [14]. Notre série a retrouvé une limite d'exérèse envahie dans 33 cas (I2\%) dont quatre avaient bénéficié d'une mastectomie et curage axillaire (MCA), une parmi les six patientes qui avait subi une quadrantectomie et deux qui étaient reçues au service en postopératoire. Il y avait une prédominance des marges saines. Ces résultats sont identiques aux études menées par Raymond et al [8] (10\%) et Chauleur et al [6] (I I,4 \% de marges d'exérèse positives).

On retient sous ce terme aussi bien les invasions des canaux lymphatiques que celles des capillaires et des veinules situées au voisinage du cancer infiltrant [13]. La présence de ces emboles vasculaires est un facteur pronostique défavorable. On peut raisonnablement penser que le plus mauvais pronostic des lésions avec emboles est à rattacher au fort pouvoir de dissémination des cellules tumorales (métastases), mais également à une sous-estimation de la taille tumorale et à une mauvaise appréciation des limites chirurgicales d'exérèse (récidive) [16].

Dans notre série, la recherche d'emboles n'était pas large, notée que chez 29 patientes ( $10 \%$ de la série) dont 18 patientes (62\%) en présentaient des emboles vasculaires.

\section{CONCLUSION}

Les facteurs pronostiques sont multiples et souvent péjoratifs chez nos patients avec une prédominance des femmes jeunes, des cancers localement avancés et des formes biologiques agressifs. Une disponibilité plus accrue de l'immunohistochimie sera bénéfique pour une meilleure prise en charge de nos patientes.

\section{CONFLITS D'INTERET}

Les auteurs n'ont déclaré aucun conflit d'intérêts.

\section{REFERENCES}

I. Dem Ahmadou, Traore B, Dieng MM, Diop PS, Ouajdi $\mathrm{T}$, Lalami $M T$, et al. Les cancers gynécologiques et mammaires à I'Institut du cancer de Dakar] Cahiers Santé. 200818 (I) : 25-28.

2. Remontet L, Esteve J, Bouvier A M et al. Incidence et mortalité des cancers en France. Période de 19802005. Rev. Epidemiol. Santé publique 2008 ; 56(3) : I59-I75.

3. Trétarre B, Guizard AV, Fontaine D, et al Cancer du sein chez la femme : incidence et mortalité, France 2000. Cancer 2004; 100(4) 715-722.

4. Kroman N, Jensen M, Wohlfahrt J, Mouridsen H T et al. Factors influencing the effect of age on prognosis in breast cancer : population based study. BMJ 2000 ; $320: 474-9$.

5. Gajdos C, Tartter PI, Bleiweiss IJ, Bodian C, Brower ST. Stage 0 to stage III breast cancer in young women. J Am coli surg 2000 ; 190 (5) : 523-9.

6. Chauleur C, Vulliez L, Trombert B et al. Facteur de risque de récidive des cancers du sein traité par 
chirurgie conservatrice : à propos de 254 cas. J. gynécologie-obstétrique et biologie de la reproduction 2008 ; 370 : 170- 178.

7. Cutuli B. Influence of locoregional irradiation on local control and survival in breast cancer. Cancer Radiothérapie 1998 ; 2(5) : 446-459.

8. Raymond V P, Pauline $\mathrm{T}$, Alexanda $\mathrm{C}$ et al. Clinicopathologie factors of the recurrent tumor predict outcome in patients with ipsilateral breast tumor recurrence. American cancer society 2011; II7(10): 2035-2043.

9. Treilleux I, Bremond A. Pronostic des cancers du sein. Encycl Méd Chir, gynécologie, 865-F-20, 2002.

10. Cuzick J, Sestak I, Baum M et al. Effect of anastrozole and tamoxifen as adjuvant treatment for early-stage breast cancer: 10 year analysis of the ATAC trial. Lancet oncol. 2010 ; I I(I2): II35-II4I.

II. Punglia R S, Saito A M, Neville B A et al. Impact of interval from breast conserving surgery to radiotherapy on local recurrence in older women with breast cancer: retrospective cohort analysis. B. M.J. 2010 ; 340 : 840-845.

12. Delozier T. Hormonothérapie du cancer du sein. Jour Gyn Obst et Bio Reprod, 2010 ; 39 (8) :7I-78.

13. Cornez N, Piccart $M$ J. Cancer du sein et Herceptin@. Bull Cancer 2000; 87 (I I): 847-58.

14. Sigal-Zafrani B. Evaluation des limites d'exérèse chirurgicale en pathologie mammaire. 32ème Journées de la SFSPM, Strasbourg, novembre 2010: 205-II.

15. Serin D, Escoute M. Cancer du sein de la femme âgée. Encycl Med chir, Gynécologie; 6894-20, 1999, 6p.

16. Arnould L. Aspects anatomopathologiques généraux des tumeurs et de leurs extensions. Implications pronostiques et thérapeutiques. Cancer/radiother 2002; 6: 61-69. 